\title{
Determining the epigenome using DNA alone
}

\author{
Coby Viner ${ }^{1,2}$ and Michael M. Hoffman ${ }^{1,2,3, *}$ \\ ${ }^{1}$ Department of Computer Science, University of Toronto, Toronto, ON, Canada \\ ${ }^{2}$ Princess Margaret Cancer Centre, Toronto, ON, Canada \\ ${ }^{3}$ Department of Medical Biophysics, University of Toronto, Toronto, ON, Canada \\ *email: michael.hoffman@utoronto.ca
}

Predicting gene regulatory activity at a genomic locus from its DNA sequence has long remained an elusive goal. In this issue of Nature Methods, Whitaker et al. ${ }^{1}$ make a giant step towards that goal. They have trained computational models that predict, with great accuracy, the locations of histone marks and DNA methylation solely from sequence data. These unprecedented results demonstrate that much of a cell's epigenomic state arises from its genomic sequence. Whitaker et al. ${ }^{1}$ identify specific DNA sequence motifs that may have an outsize effect on the appearance of particular epigenomic marks, both generally and within specific cell types.

A single organism's cells mostly share the same genomic sequence, but different cell types show dramatic differences in gene expression. Each cell marks activation and repression of transcription at specific genomic loci using epigenomic factors. These factors include methylation of the DNA itself and covalent modifications of the histone proteins that organize DNA into chromatin. The most studied histone modifications are methylation and acetylation of lysine residues on core histone H3. These modifications have roles in propagating cellular identity, ${ }^{2}$ activating and repressing transcription, ${ }^{3}$ and marking important gene regulatory features like transcription start sites and enhancers. ${ }^{4,5,6}$ Here, we refer to the map of locations of all histone modifications and DNA methylation as the cell's epigenomic state.

Cells of a particular type, such as human mesenchymal stem cells, converge during development to an epigenomic state similar to other cells of that type. To reproduce a cell-type-specific characteristic epigenomic state at any locus, the cell has at its disposal two information sources. Those sources are the previous, somewhat transient, epigenomic properties of that locus, and the more durable DNA sequence. Given that the cell reliably reproduces its epigenome during development using only this information, it should be possible to use the same information to predict much of the epigenomic state computationally. This theoretical possibility has not been realized substantively until now.

Whitaker et al. ${ }^{1}$ are among the first to show substantial prediction of epigenomic state from a small number of DNA motifs. They developed a software application, Epigram, to predict DNA methylation and histone modification from sequence motifs. They characterize motifs by their association with particular epigenomic marks in particular cell types. Also, they show that some of these motifs tend to be found specifically at either the centers or edges of regions of epigenomic mark enrichment.

When submitted, this work was the first to directly demonstrate substantial predictability of epigenomic modifications from DNA sequence alone. Previous work correlated motif disruption with variation in $\mathrm{H} 3$ lysine 27 acetylation (H3K27ac), ${ }^{7}$ but Epigram can explain more variation using fewer motifs. Another recent article by Benveniste et al. ${ }^{8}$ also uses sequence information to predict epigenomic state. While Epigram extracted more predictive information from sequence alone, Benveniste $\mathrm{et} \mathrm{al.}{ }^{8}$ performed almost as well. Benveniste $e t a l .{ }^{8}$, however, slightly surpassed Epigram's performance using chromatin immunoprecipitation sequencing (ChIP-seq) data on transcription factor presence. Since many of the motifs found by Epigram to have predictive value are similar to 
published transcription factor binding motifs, both studies suggest a major role of known transcription factors in driving epigenomic state. Nonetheless, predicting epigenomic state without relying on any site-specific biochemical data (such as ChIP-seq data) is more impressive. Such predictions can be applied even when the additional biochemical data does not exist.

What sets Epigram apart from previous approaches is the careful and thorough removal of several confounding factors. Whitaker et al. ${ }^{1}$ use a random forest ${ }^{9}$ classifier to learn the difference between sequences found near regions enriched for an epigenomic mark (foreground) and sequences that aren't (background). Before doing this, however, Epigram uses a sequence-set balancing procedure to ensure that the sequences in the foreground and background sets have similar lengths and $\mathrm{G}+\mathrm{C}$ contents. This reduces the risk of learning a classification based primarily on those simple features rather than sequence motifs. In a separate step, the Epigram pipeline also adjusts for $\mathrm{G}+\mathrm{C}$ content bias in sequencing.

While Whitaker et al. ${ }^{1}$ identify sequence motifs associated with epigenomic state, they do not demonstrate that these motifs cause the deposition of epigenomic properties. One could show that the identified motifs are essential for writing the marks in question using genome editing technologies ${ }^{10}$ or other laboratory techniques (Figure 1). This paper opens the way to those studies and other mechanistic investigations of the driving forces behind epigenomic state.

\section{References}

1. Whitaker, J. W., Chen, Z. \& Wang, W. Predicting the human epigenome from DNA motifs. Nat. Methods, 1-15 (Sept. 2014).

2. Campos, E. I., Stafford, J. M. \& Reinberg, D. Epigenetic inheritance: histone bookmarks across generations. Trends Cell Biol. 24, 664-674 (Sept. 2014).

3. Kouzarides, T. Chromatin modifications and their function. Cell 128, 693-705 (Feb. 2007).

4. Liang, G. et al. Distinct localization of histone H3 acetylation and H3-K4 methylation to the transcription start sites in the human genome. Proc. Natl. Acad. Sci. U.S.A. 101, 7357-62 (May 2004).

5. Heintzman, N. D. et al. Distinct and predictive chromatin signatures of transcriptional promoters and enhancers in the human genome. Nat. Genet. 39, 311-8 (Mar. 2007).

6. Zhou, V. W., Goren, A. \& Bernstein, B. E. Charting histone modifications and the functional organization of mammalian genomes. Nat. Rev. Genet. 12, 7-18 (Jan. 2011).

7. Kasowski, M. et al. Extensive variation in chromatin states across humans. Science 342, 750-2 (Nov. 2013).

8. Benveniste, D., Sonntag, H.-J., Sanguinetti, G. \& Sproul, D. Transcription factor binding predicts histone modifications in human cell lines. Proc. Natl. Acad. Sci. U.S.A. 111, 13367-72 (Sept. 2014).

9. Breiman, L. Random forests. Mach. Learn. 45, 5-32 (2001).

10. Hou, Z. et al. Efficient genome engineering in human pluripotent stem cells using Cas9 from Neisseria meningitidis. Proc. Natl. Acad. Sci. U.S.A. 110, 15644-9 (2013). 


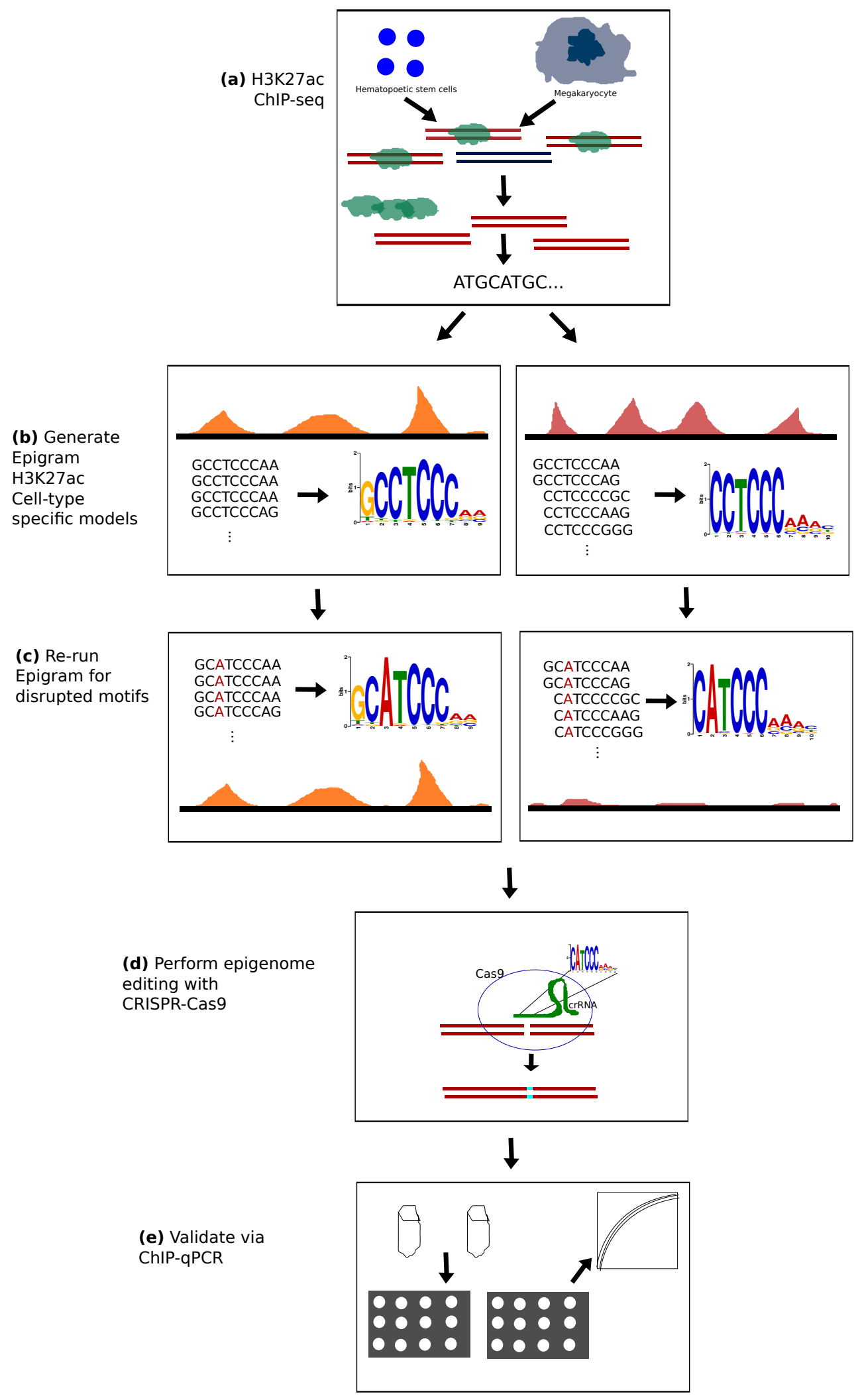

Figure 1: Potential experiment for cell-type-specific epigenome editing. (a) Collect H3K27ac ChIPseq data in both hematopoetic stem cells and megakaryocytes. (b) Learn an Epigram model for each cell type. (c) Run Epigram using learned models and modified sequences which cause motif disruption. Find modified sequences that primarily affect one cell type, not the other. (d) Use CRISPR-Cas9 to edit genome sequences. (e) Validate outcome via ChIP-quantitative polymerase chain reaction (qPCR). 